\title{
A Golgi stain for dendritic function?
}

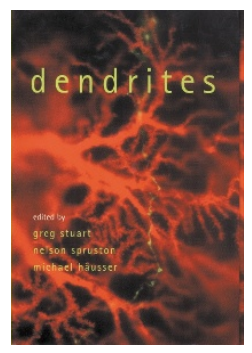

\section{Dendrites}

Edited by Greg Stuart, Nelson Spruston

and Michael Hausser

Oxford University Press, 1999. $\$ 55.00$

hardcover, pp 369

ISBN 0-19-850488-8

Reviewed by Kevan Martin

Dendrites are among the most extraordinary and visually compelling structures in the nervous system. Purkinje observed them as early as 1837 , and Deiters beautifully depicted them in his drawings of individual dissected motoneurons in 1865 . Wilhelm His coined the name 'dendrite' in 1890, only 7 years before Charles Sherrington first used the term 'synapse'. By then Camillo Golgi had invented his eponymous stain, which casts in exquisite silver sculptures the finest ramifications and processes of neurons. Golgi's discovery enabled his rival, Santiago Ramon y Cajal, to define neuronal circuits of retina, cerebellum and spinal cord, based on the inspired assumption that information was transferred from dendrites to the cell body and thence down the axon to synapses on dendrites. Thus, from the earliest decades of modern neuroanatomy and neurophysiology, dendrites and synapses were intimately linked. They remain so today, agree the authors of Dendrites, a cross-section of the state of the art in 14 chapters contributed by 19 authors.

Why mention these ancient observations when reviewing a book about work using the latest techniques_-patch-clamp recordings, optical imaging of ion concentrations and molecular probing of proteins and RNAs-which promise to revolutionize the study of dendrites? The answer is that chapter after fascinating chapter makes it evident that nothing on the horizon, either technically or conceptually, will solve the mysteries of dendritic function in the way that a single technique, Golgi's stain, did for structure.

These early structural studies first raised the central question repeated here by the editors: why is dendritic structure so enormously variable? The equally long-standing speculation is that this variability is fundamental to dendritic function. If so, then we can expect no unified view of den-

Kevan Martin is at the University/ETH Zurich, Institute of Neuroinformatics,

Winterthurerstr. 190, 8057 Zurich, Switzerland. e-mail:kevan@ini.phys.ethz.ch drites to emerge, because the particularities of the network containing the dendrites will determine their structure and function. Thus unless we understand the network, we should not expect to understand the functions of particular dendrites, let alone the reasons for the wide variety of dendritic structures. We should not expect to define a canonical dendrite, a single representative of all the varieties so far described, or suppose that results found for one dendrite are necessarily generalizable. The new techniques also evidently mean that relatively little of the structural heterogeneity will be investigated at a functional, biochemical or molecular level. Already most explorations of dendrites, experimental or theoretical, are of particular dendrites of particular neuronal subtypes in just three brain regions. Ironically, we understand vastly more of the varieties of function of the products of the worm, fly and human genome than we do of the function of the varieties of neuronal dendrites that we suppose to be so fundamental in cognition. In the next decades, we will surely discover a great deal about the properties of apical dendrites of pyramidal cells in hippocampus and in neocortex, and of the elaborate dendrites of cerebellar Purkinje cells, but the function of the vast majority of structurally distinct dendrites will (sadly) remain unexplored.

One particularly eye-catching structure appears frequently in Dendrites' illustrations, a miniscule process that bristles in multiple forms from the surface of many dendrites. The reality of these dendritic spines, a few microns in size, was initially in doubt. They could be artifacts, "the result of tumultuous precipitation of the silver" wrote Ramon y Cajal in his first report of his work with Golgi's new stain, but their constancy from experiment to experiment, "inclines us to judge that this is the normal condition." The electron microscope allowed later generations to confirm Ramon y Cajal's insight that spines were the specialized sites for synapses. Spines contribute half or more of the surface area of spiny dendrites, and most excitatory synapses are formed with them. Thus, spines are a point of convergent interests of synaptic physiologists, anatomists, neurochemists, molecular neurobiologists and theoreticians. A variety of roles in development, plasticity and computation have been proposed for them, but their functions remain a mystery. Recent technical advances in light microscopy, however, have enabled scientists to observe the growth of new spines in response to changes in dendritic activation, offering a new means of relating dendritic microstructure to learning and memory.

Most of what we now think about the function of dendrites arises not from experimental observations, but from theory. This makes dendrites special, as there are few other areas of neuroscience where experimentalists must rely so much on theoretical work. It is thus fitting that, although the aims and future interests of its many younger contributors are well represented, Wilfrid Rall's contribution, at age 77, lies at the center of Dendrites, both literally and intellectually. His chapter is delightful, not only because it links the generations, but also because he was the source of many modern ideas of dendritic function. Rall's application of cable theory to neurons in the late 1950s fundamentally revised physiologists' view of dendrites. Rall worked with and inspired generations of physiologists and theoreticians, many of whom contribute directly or indirectly to Dendrites. These researchers have now laid out a basic framework for interpreting physiological data, which may be the nearest we ever get to a Golgi stain for dendritic function.

The editors sum up four key areas for future development. The fourth is surely the most important: "a better understanding of the relationship between individual features of dendrites and higher-level function." In 1943, McCulloch and Pitts discovered that extraordinary computational richness can arise from networks of simple linear threshold neurons without dendrites. The Purkinje cell illustrated on the dust jacket begs us to explain what computational advantages accrue by adding dendrites. The truth is we cannot yet answer the simple question of whether dendritic geometry, together with a given synaptic input, implements specific computations, or whether the specific locations of synapses are largely irrelevant, and dendrites exist in a variety of forms simply to connect to a similar variety of incoming axon geometries. Dendrites certainly receive synaptic inputs, but the question remains, do they really process them? Many of the authors of Dendrites intend to find out. 